\title{
In Vivo and In Vitro Cross Neutralization Studies of Local Rabies Virus Isolates with ERA Based Cell Culture Anti-Rabies Vaccine Produced In Ethiopia
}

Abebe Mengesha Aga*, Yalemtsehay Mekonnen ${ }^{2}$, Birhanu Hurisa ${ }^{1}$, Tihitina Tesfaye ${ }^{1}$, Hailu Lemma ${ }^{1}$, Gezahegn Kebede ${ }^{1}$ Amha Kebede ${ }^{1}$, Dereje Niguse ${ }^{1}$, Gashaw G/Wold ${ }^{1}$ and Kelbessa Urga ${ }^{1}$

${ }^{1}$ Ethiopian Public Health Institute, Ethiopia

${ }^{2}$ Addis Ababa University, Ethiopia

*Corresponding author: Abebe Mengesha, Ethiopian Public Health Institute, Ethiopia, Tel: +251112134032; E-mail: agagurmu@yahoo.com (or) abebem@ephi.gov.et Received date: 17 September 2014; Accepted date: 24 October 2014; Published date: 27 October 2014

Copyright: (c) 2014 Aga, et al. This is an open-access article distributed under the terms of the Creative Commons Attribution License, which permits unrestricted use, distribution, and reproduction in any medium, provided the original author and source are credited.

\begin{abstract}
Rabies is a worldwide problem, and the case is most severe in developing countries where cell culture anti-rabies vaccines are unaffordable or the available nervous tissue-derived vaccines are of questionable immunogenicity and may cause neurological complications. The aim of this research was to study cross protection of local rabies virus isolates with Evenyl Roktincki Abelseth (ERA) based cell culture anti-rabies vaccine produced in Ethiopia and to develop challenge virus from local isolates. The viruses were isolated from rabid dogs' brains and human saliva, and adapted on Swiss albino mice and cell lines. Cross protection with ERA based vaccine was studied by in vivo and in vitro methods. For in vivo method, a group of mice were immunized on day zero and seven with $0.5 \mathrm{ml}$ (1:5 dilutions) of ERA based cell culture anti-rabies vaccine produced locally. On day fourteenth, mice were challenged with working dilution of each local isolates and one group with challenge virus standard (CVS-11), and observed for further 14 days. High protection was recorded in CVS-11 challenged mice and low protection in all local isolates $(p=0.045)$; specifically protection to HOS challenged mice was very low. In vitro test was done by fluorescent antibody virus neutralization (FAVN) test on BHK-21 cell lines. Sera from dog immunized with locally produced vaccine and OIE serum were incubated with local virus isolates and CVS-11 for 48 hours in the presence of cell lines. Maximum antibody titer $(2.74 \mathrm{lU} / \mathrm{ml})$ was obtained with CVS-11 challenge virus and minimum antibody titer $(1.55 \mathrm{lU} / \mathrm{ml})$ was obtained with cow origin $(\mathrm{CO})$ virus isolate. All locally isolated rabies virus show low antibody titer when compared to CVS-11 and PV-12 $(p=0.000)$. From the results, it can be concluded that local isolates have some genetic variation from fixed virus strain which can affect efficacy of the candidate vaccine and potency value should be set in-terms of local isolate using as challenge virus. Generally, the exact genetic relationship should be studied by molecular techniques and locally isolated virus should be used as challenge virus for vaccine quality control.
\end{abstract}

Keywords: Cell culture vaccine; Cross protection; Local virus isolate; Rabies

\section{Background}

Rabies is a viral disease that affects the central nervous system (CNS) of mammals and has an extremely high case fatality rate. In developing countries, with limited access to high-quality anti-rabies biologics, approximately 55,000 people and millions of animals die every year due to rabies [1]. Rabies is endemic in developing countries of Africa and Asia, and most human deaths occur in these endemic countries [2]. The annual cost of rabies in Africa and Asia was estimated at US \$ 583.5 million, most of which is due to cost of post exposure prophylaxis [3]. Ethiopia being one of the developing countries is highly endemic for rabies.

In Ethiopia, rabies is an important disease that has been recognized for many centuries. The incidence of human post exposure treatments and human rabies cases per million population of Ethiopia were 73.6 and 12.6, respectively [4]. In Africa, the highest recorded human death due to rabies for the year 1998 was 43 which were reported from Ethiopia [5]. Most of the cases are due to bite by stray dogs. The dog is the species most responsible for human exposure, with over $94.01 \%$ of the total positive animals [6]. As a result, vaccination of domestic animals, mostly dogs and wild animals, mostly foxes are the only way to control and eliminate rabies [7]. Passive reports usually underestimate incidence and are poor indicator of the status of the disease in countries like Ethiopia where human and animal health information systems are inadequate. There is lack of accurate and quantitative information on rabies both in humans and animals, and little is known about the awareness of the people about the disease to apply effective control measures in Ethiopia.

Pre- or post-exposure prophylaxis, using vaccination and passive immunoglobulin administration is the only effective way to prevent rabies after infection with a lyssavirus [8]. The efficacy of both active and passive immunization is likely to be affected by antigenic differences between related lyssaviruses. Closely related lyssaviruses circulate among bats, and can cause an illness identical to rabies in people and domesticated animals with some only relatively different genetic makeup [9]. Rabies vaccines and post-exposure prophylaxis are thought to provide some protection against some of these viruses (Phylogroup I), but not some of phylogroup II genotypes [10].

Rabies-related lyssaviruses can be found even in countries classified as rabies free. The virus has a non-segmented and negative-stranded RNA genome with about 12,000 nucleotides. Lagos bat virus, Duvenhage virus, European bat lyssavirus (EBLV) 1, EBLV 2, Australian bat lyssavirus (ABLV), Mokola virus and Irkut virus have caused clinical cases in humans or domesticated animals through host 
Citation: $\quad$ Aga AM, Mekonnen Y, Hurisa B, Tesfaye T, Lemma H, et al. (2014) In Vivo and In Vitro Cross Neutralization Studies of Local Rabies Virus Isolates with ERA Based Cell Culture Anti-Rabies Vaccine Produced In Ethiopia. J Vaccines Vaccin 5: 256. doi: $10.4172 / 2157-7560.1000256$

Page 2 of 5

switching. Rabies virus and the rabies-related lyssaviruses have been classified into two or more phylogroups, based on their genetic relatedness. Phylogroup I contains rabies virus, Duvenhage virus, EBLV 1, EBLV 2 and Australian bat lyssavirus, and Phylogroup II consists of Lagos bat virus, Mokola virus and Shimoni bat virus [11]. Viruses that are more closely related to rabies virus can be neutralized, at least to some extent, by antibodies to rabies virus [9]. Viruses that are far from rabies virus in their glycoprotein genetic makeup cannot be neutralized by antibody raised to classical rabies virus and can affect efficacy of the vaccine [11,12]. In Ethiopia, brain samples collected from domestic animals, two rabies-related viruses were isolated [13]. According to their reactivity pattern with anti-nucleocapsid monoclonal antibodies, they were characterized as Lagos bat virus and Mokola virus. But, little is known about the biological characteristics of these isolates and about the ability of current rabies vaccines to elicit immune responses which would provide cross-protection.

The potency test for rabies vaccine was originally developed at the National Institute of Health (NIH), Bethesda, USA. This test measures the degree of protection conferred by inactivated vaccines in immunized mice challenged with rabies virus [14]. There appears to be three important considerations in assessing a potency test before the release of the vaccine [15]. From these, the third requirement states that standardization of different vaccines will be comparable in relation to challenge virus strain used from circulating street rabies virus from the area of vaccine to be applied.

In Ethiopia, many people receive anti-rabies post exposure treatments annually due to wide-spread of dog bites. The isolation of Mokola and Lagos bat viruses from domestic animals in Ethiopia [13] is of public and veterinary concern due to lack of effective vaccines against these agents which cannot be protected by vaccine from fixed rabies virus strains. Studies done in France confirm that $14.7 \%$ divergence between wild type rabies virus and vaccinal strain which can strongly affect efficacy and potency of anti-rabies vaccine [15].

Since the 1940's, Ethiopia produces and uses Fermi-type anti-rabies vaccine which have low immunogenicity and can cause complication. Currently Ethiopian Public Health Institute (EPHI) has successfully produced cell culture anti-rabies vaccine from PV and ERA fixed rabies virus strain [16]. ERA virus strain was selected to produce antirabies vaccine locally based on its multiplicity titer for mass production compared to $\mathrm{PV}$ virus strain. The efficacy of this vaccine should be tested with local rabies virus isolates for cross protection compared to CVS-11 challenge virus strain.

\section{Materials and Methods}

\section{Laboratory animals}

Swiss albino mice were used for clinical isolation, blind passage, in vivo virus titer determination and mice protection study. Mice, 3-4 weeks old, weighing 6-8 grams with identical sex were used. Two dogs immunized with locally produced vaccine were used as source of sera used for in vitro cross neutralization test. All mice were obtained from EPHI, Laboratory Animal Breading Center. This research was performed after obtaining ethical clearance from EPHI scientific and ethical clearance committee on laboratory animal care and usage throughout the experiment.

\section{Cell lines}

A Baby Hamster Kidney (BHK-21) cell lines purchased and supplied from American Type Culture Collection (ATCC) was used for the adaptation of the virus isolates, in vitro cross neutralization test and virus titer determinations.

\section{Vaccine}

ERA fixed vaccinal strain based anti-rabies vaccine was produced by propagating the virus on Vero cell lines. The virus titer was greater than $10^{6} \mathrm{TCID}_{50} / \mathrm{ml}$ before inactivation [16]. Safety and potency test was done after purification by centrifugation. Centrifugation was done at $5,000 \mathrm{rpm},\left(\right.$ at $\left.4^{\circ} \mathrm{C}\right)$ for 15 minutes to separate viral antigen and cell debris. This vaccine was used for mice immunization during challenge test with local isolate and CVS-11. Dogs were also immunized with this vaccine and sera were collected for in vitro cross neutralization test.

\section{Virus used}

Samples were collected from rabid animal brain and human saliva for this study. A total of four local isolates were used. Four local virus isolates; two from human saliva (human origin Wollega (HOW) and human origin Sululta (HOS)), one from rabid dog brain (dog origin (DO) from Gojjam) and one from rabid cow brain (cow origin (CO) from Butajira) were used (Table 1).

\begin{tabular}{|l|l|l|l|}
\hline $\begin{array}{l}\text { Type of } \\
\text { Virus }\end{array}$ & Origin & Location & Remarks \\
\hline HOW & Human Saliva & Wollega & Mice inoculation positive \\
\hline HOS & Human saliva & Sululta & Mice inoculation positive \\
\hline DO & Dog brain & Gojam & FAT positive \\
\hline CO & Cow brain & Butajira & FAT positive \\
\hline
\end{tabular}

Table 1: Type of virus used and their origin.

Pasteur Virus (PV-12) currently used for Fermi type vaccine production was used to compare neutralization of anti-rabies sera raised with ERA fixed virus strain based cell culture anti-rabies vaccine. Challenge virus standard (CVS-11) was used as standard for comparison of protection in comparison to local virus isolates.

\section{Virus preparation}

Fluorescent antibody test (FAT) was used to test presence of virus in the sample collected before adaptation to mice brain and cell lines. Brain sample was applied on slide and fixed in acetone at $-20^{\circ} \mathrm{C}$ for 5 minute. Fluorescent isothiocyanate labelled anti-lyssavirus antibodies originated from Russia were used as monoclonal antibody which recognizes glycoprotein of lyssavirus. After the acetone dried out, samples were covered with FITC anti-rabies antibody and incubated at $37^{\circ} \mathrm{C}$ for 30 minutes. Slides were rinsed with PBS twice and examined under 40x objective fluorescent microscope. The virus was adapted to mice brain by passaging five times on mice brain without finding virus titer. After five passages, the last brain samples were homogenized in $10 \% \mathrm{w} / \mathrm{v}$ PBS, and purified by centrifugation (at 3,000 $\times \mathrm{g}$ ) and filtered with $0.22 \mu \mathrm{m}$ sterile filter. BHK-21 cell lines were cultured in $75 \mathrm{~cm} 2$ tissue culture flask using Eagle Minimum Essential Medium (EMEM) supplemented with $10 \%$ fetal bovine serum. After 72 hours, when 
Citation: $\quad$ Aga AM, Mekonnen Y, Hurisa B, Tesfaye T, Lemma H, et al. (2014) In Vivo and In Vitro Cross Neutralization Studies of Local Rabies Virus Isolates with ERA Based Cell Culture Anti-Rabies Vaccine Produced In Ethiopia. J Vaccines Vaccin 5: 256. doi: $10.4172 / 2157-7560.1000256$

Page 3 of 5

monolayer (cell confluence) was reached, cells were trypsinized. The cell concentration was determined and inoculated with brain supernatant at 0.1 virus/cells. Infected cells were incubated at $37^{\circ} \mathrm{C}$ for 30 minutes with mixing every 5 minutes. After 30 minutes, cells were centrifuged at $2500 \mathrm{rpm}$ for 10 minutes, supernatant removed and cell pellet re-suspended in $5 \mathrm{ml}$ complete medium (contain 10\% fetal bovine serum, 3.5\% $\mathrm{NaHCO}_{3}$ and $1 \%$ L-glutamine). The cell suspensions were transferred to $25 \mathrm{~cm}^{2}$ tissue culture flasks and incubated for 72 hours at $37^{\circ} \mathrm{C}$ in a humid incubator with $5 \% \mathrm{CO}_{2}$. Viruses were passaged twenty times on cell lines with titration every five passage. At each passage, tissue culture supernatant (TCSN) were harvested, aliquoted, and stored at $-80^{\circ} \mathrm{C}$. Titration of the virus was used to determine growth and multiplicity infection of the rabies virus during passage on mice brain and cell lines.

\section{Anti-sera}

Dogs were immunized with $1 \mathrm{ml}(2.5 \mathrm{IU} / \mathrm{ml})$ of locally produced ERA based anti-rabies vaccine subcutaneously. Blood drawn and sera were collected at day 120 from two dogs immunized with ERA fixed rabies virus based cell culture vaccine produced locally. The serum was heat inactivated at $56^{\circ} \mathrm{C}$ for 30 minutes and stored at $-20^{\circ} \mathrm{C}$ until use. For positive control serum, canine based standard serum supplied by OIE was used. Negative dog sera were used as negative control as a quality control.

\section{Mouse protection study}

Mice protection test is an in vivo method used to measure protection of mice immunized with a candidate vaccine when challenged with challenge virus. For the immunization of mice, locally produced anti-rabies vaccine (ERA strain based), propagated in Vero cell lines and chemically inactivated with formalin was used. The vaccine potency was determined previously using NIH potency test protocol and greater than $2.5 \mathrm{IU} / \mathrm{ml}$ potency value obtained. In mice protection test, five groups of mice containing 16 replicate in each group were immunized intraperitoneally with $0.5 \mathrm{ml}$ (1:5 dilutions) of ERA based vaccine twice on day zero and day seven. The mouse intracerebral lethal dose (50MICLD50/0.03 ml) was determined by injecting $0.03 \mathrm{ml}$ of all virus isolates and challenge virus standard (CVS-11) on four week-old mice before challenge day. On day 14 after first immunization, all group of mice and control groups were challenged with working dilution of local rabies virus isolates and CVS-11 intracerebrally. The mice were observed for 14 days after challenge inoculation. All specific and non-specific death was recorded separately. Mice dead after five days without showing any sign of rabies was confirmed by FAT test on brain sample. Protection index of candidate vaccine in mice challenged by local virus isolates was determined based on the survival rate of the challenged mice when compared to challenge virus standard.

\section{Cross neutralization (in vitro)}

The neutralizing abilities of the sera raised to ERA fixed rabies virus strain based vaccine were assessed using fluorescent antibody virus neutralization (FAVN) tests on BHK-21 cell lines. Volumes of viruses' tissue culture infectivity dose (50TCID50/50 $\mu$ l) were added to serial three fold dilutions of test and reference serum on 96 well microtitration plates with four replicate. The plate was incubated for 48 hours at $37^{\circ} \mathrm{C}$ with $5 \% \mathrm{CO}_{2}$ in the presence of BHK-21 cell lines. After 48 hours, the plate was stained with anti-rabies antibody labeled containing fluorescent conjugate. The reading was done as positive/ negative fluorescent apple green under fluorescent microscope. The $50 \%$ endpoint dilution, where neutralization of the virus ceased, was calculated using Spearman Karber methods as shown below.

$$
\text { Serum titer }(I U / \mathrm{m} I)=\frac{[(10(\text { serum } \log D 50 \text { value })) \times \text { theoreticaltiterofOIEs }}{(10(\log D 50 \text { ofOIEserum } 0.5 \mathrm{IU} / \mathrm{mI}))}
$$

\section{Results}

\section{Virus adaptation result}

Titer of the virus increase throughout blind passage and show maximum of virus titer after twenty passages on BHK-21 cell lines. In each blind passage, the virus shows different multiplicity of infection by in vitro titration methods at each blind passage.

\begin{tabular}{|l|l|}
\hline Virus origin & MICLD $_{50} / \mathbf{0 . 0 3} \mathbf{~ m l ~ ( P 2 0 )}$ \\
\hline HOW & $10^{4.5}$ \\
\hline HOS & $10^{5.9}$ \\
\hline DO & $10^{5.5}$ \\
\hline CO & $10^{4.5}$ \\
\hline PV-12 & $10^{5.25}$ \\
\hline
\end{tabular}

Table 2: Virus titration result (in vivo).

According to these results, the rabies viruses showed increase infectivity titer. After twenty passages on cell lines, in vivo virus titer were performed (Table 2) and this can be used for challenge test for mice protection study after titer adjustment.

\section{Mice protection test}

Mice protection study was used to evaluate protection of mice immunized with ERA fixed virus strain based vaccine when challenged with local isolates by in vivo method. In this test, mice showed different incubation period when compared to fixed challenge virus strain. A minimum of four days difference was observed with five days additional incubation period to complete the test in addition to fourteen days follow up. The result shown in Figure 1 summarizes death/survival rate of mice after challenged with local rabies virus isolates and challenge virus standards.

According to the results, locally produced ERA based anti-rabies vaccine showed different level of protection against each local virus isolates and challenge virus standard. Eventhough the viral concentration of each virus was adjusted to 50MICLD50/0.03 ml before challenge, the number of mice protected by locally produced ERA based vaccine was different. The highest protection was observed for CVS-11 challenge virus standard with eleven mice survived out of sixteen. For all control groups, eight mice inoculated per virus and minimum of six death $(75 \%)$ were recorded which was greater than $50 \%$. The minimum protection index was observed with human origin Sululta (HOS) local virus isolate with nine deaths out of sixteen mice challenged. Data analysis performed using Fisher's exact test and linear by linear association showed significance difference $(p=0.046)$ for CVS-11 challenged mice and local virus isolates. But there was no significance difference in protection between local isolates. The other local virus isolates (HOW, DO and $\mathrm{CO}$ ) showed low protection when compared to challenge virus standard. 
Citation: $\quad$ Aga AM, Mekonnen Y, Hurisa B, Tesfaye T, Lemma H, et al. (2014) In Vivo and In Vitro Cross Neutralization Studies of Local Rabies Virus Isolates with ERA Based Cell Culture Anti-Rabies Vaccine Produced In Ethiopia. J Vaccines Vaccin 5: 256. doi: $10.4172 / 2157-7560.1000256$

Page 4 of 5

\section{Cross neutralization test}

Cross neutralization test was performed to evaluate neutralizing level of local virus isolates with antibody raised to ERA fixed rabies virus strain. Anti-sera of two dogs (D1 and D2) previously immunized with ERA based anti-rabies vaccine produced locally were used to neutralize the viruses of local origin (HOW, HOS, DO and CO) and CVS-11 challenge virus standard. PV-12 vaccinal strain was used to compare neutralizing capacity between fixed virus strain (PV-12 and CVS-11) and clinical rabies virus isolates. All local virus isolates were positive to lyssavirus monoclonal antibody. Based on the FAVN test results, different titers of dogs (D1 and D2) and OIE anti-rabies antibody were obtained for each local virus isolate and CVS-11 challenge virus standard (Figure 2).

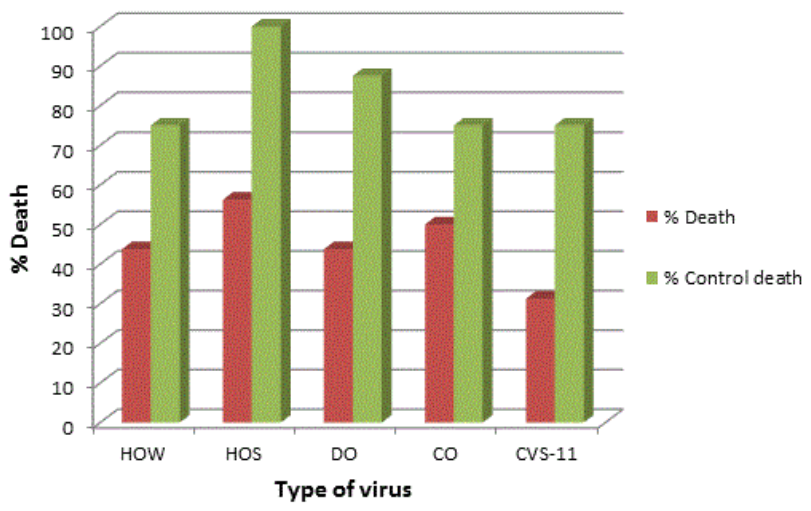

Figure 1: In vivo mice protection study results showing survival and death rate of immunized mice when challenged with challenge virus standard and local virus isolates.

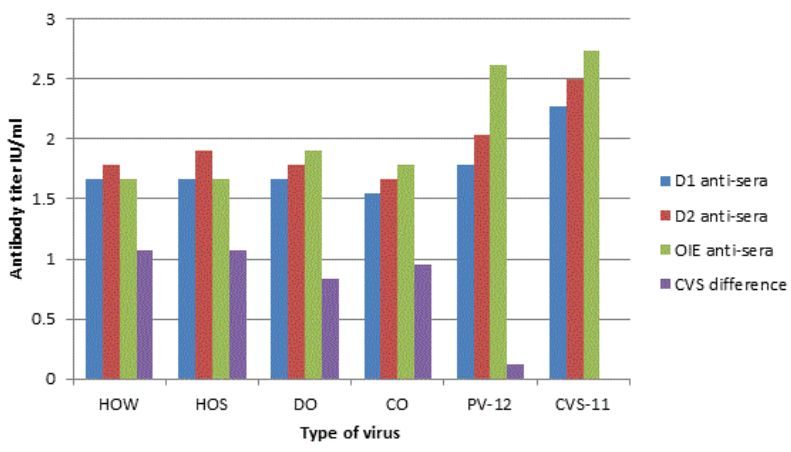

Figure 2: In vitro cross neutralization test result of challenge virus standard and local virus isolates with antibody raised to locally produced vaccine, $\mathrm{D} 1=\operatorname{dog} 1$ sera; $\mathrm{D} 2=\operatorname{dog} 2$ sera.

According to the data analysis performed using Fisher's exact test, cross tabulation showed significance difference in antibody titer between challenge virus standard and local rabies virus isolates $(\mathrm{p}<0.05)$. But, there was no statistical significance difference between sera at each virus isolate. The results indicate that neutralization of local virus isolates with dogs (D1 and D2) and OIE anti-sera was lower when compared to standard challenge virus strain and PV-12 fixed rabies virus strain. Antibody titer was highest for all sera when fixed virus strain (PV-12 and CVS-11) used for neutralization compared to local isolates. The difference in serum antibody titer when local virus isolate used were very low $(0.36 \mathrm{IU} / \mathrm{ml})$. But, the difference in antibody titer between local isolates and fixed virus standard was very high (1.19 $\mathrm{IU} / \mathrm{ml}$ ). Each dog sera also showed some difference in serum antibody titer for the same local virus isolate, challenge virus standard and PV-12 vaccinal strain, but the difference was very low $(0.25 \mathrm{IU} / \mathrm{ml})$ ( $>0.05$ ) (Figure 2). Antibody titers for dogs as well as OIE were highest when PV-12 was used as challenge virus in comparison to local isolates. Based on the history of fixed virus strain development, PV-12 and CVS-11 originated from the same virus origin. The only difference was the number of passages and host used for adaptation mechanisms. Several passages may bring genetic mutation but virulence of the virus other than cell lines and intracerebral inoculation almost declined.

\section{Discussion and Conclusion}

Cross neutralization of the viruses with antibody raised to locally produced cell culture vaccine was carried out in two ways, mice immunization and challenging (in vivo) and FAVN test (in vitro) on dog sera immunized with the locally produced ERA based cell culture vaccine. In vivo method showed different protection of immunized mice for each virus isolates and challenge virus standard (CVS-11). Even though the virus titer adjusted to 50MICLD50/0.03 ml for all virus isolates and challenge virus standard, CVS-11 challenged mice showed high protection; whereas local rabies virus isolates (HOS) showed the lowest protection. For HOS local virus isolate, only seven mice survived the challenge (43.75\%) out of sixteen which showed low protection but, CVS-11 challenge virus standard show eleven survival $(68.75 \%)$ with highest protection. The difference in protection of challenge virus standard was at least $12.50 \%$ greater than local rabies virus isolates. This support the result obtained by Wright and his coworker which state that cross-neutralization tests using sera from RABV-vaccinated humans and animals on pseudotypes with CVS-11, EBLV-1 and EBLV-2 envelopes showed that the relative neutralization titers correlated broadly with the degree of G-protein diversity [9]. According to the calculation of relative potency for a given vaccine, such difference in death/survival rate strongly affects potency result and should be carefully evaluated with local isolates to prevent the possible failure of the vaccine. These differences in protection index indicate the effect of challenge virus on potency of a given vaccine in terms of local isolates which correlate with studies done by Badrane et al. indicating that different phylogroups shows varying level of protection [17]. Studies done in USA also support this finding that neither pre-exposure vaccination nor conventional post-exposure prophylaxis using classical rabies based vaccine provided significant protection to rabies related viruses [18]. But, still the locally produced ERA based anti-rabies vaccine was protective against the local rabies virus isolates although the protection was lower when compared to CVS-11 standard challenge virus. This low protection index causes low potency value for the locally produced ERA based anti-rabies vaccine which can result in failure of protection.

During in vitro cross neutralization test, the two dog sera and OIE sera showed different antibody titer for each virus. The highest antibody titer was recorded for OIE anti-sera $(2.74 \mathrm{IU} / \mathrm{ml}$ and 2.62 $\mathrm{IU} / \mathrm{ml}$ ) when CVS-11 and PV-12 (same origin with ERA) was used as challenge virus, respectively. The lowest antibody titer was recorded for dog one (D1) $(1.55 \mathrm{IU} / \mathrm{ml})$ when cow origin virus isolate was used 
Citation: $\quad$ Aga AM, Mekonnen Y, Hurisa B, Tesfaye T, Lemma H, et al. (2014) In Vivo and In Vitro Cross Neutralization Studies of Local Rabies Virus Isolates with ERA Based Cell Culture Anti-Rabies Vaccine Produced In Ethiopia. J Vaccines Vaccin 5: 256. doi: $10.4172 / 2157-7560.1000256$

Page 5 of 5

as challenge virus. The levels of antibody titers for all test sera were above WHO recommendation $(0.5 \mathrm{IU} / \mathrm{ml})$, but the vaccine used for dog immunization have potency value of greater than $2.5 \mathrm{IU} / \mathrm{ml}$ which may improve efficacy of the vaccine resulting in high antibody production. For the three different anti-sera, the values of antibody titers were highest when CVS-11 challenge virus standard and PV-12 vaccinal strains were used compared to the local virus isolates $(\mathrm{p}<0.05)$. Studies done by Wright and his colleague support this idea that human serum samples (fixed virus strain based) neutralized CVS-11 to the greatest degree, compared to EBLV-2 and EBLV-1 and the same neutralizing profile was observed [9]. Studies done by Guo and his coworkers also support the idea of growth in virus titer bring genetic mutation and increase efficacy of the vaccine related to the adapted virus. This may indicate genetic compatibility between PV-12 and ERA fixed vaccinal strain due to their origin and several passages on different route of inoculation. Similar mechanism was stated by Sacramento and his co-workers that the wild isolate showed marked divergence from those of fixed vaccinal strains [15]. In vivo protection test also showed similar result with in vitro test which can predict the effect of challenge virus on the potency of locally produced cell culture anti-rabies vaccine. Since small numbers of mice survived local virus isolate challenge ( $25 \%$ difference) compared to CVS-11, this has significant effect on the potency of the vaccine $(p<0.05)$. Therefore, genetic origin and several passages between fixed virus strains may be the reason for compatibility in neutralization of the virus with antibody raised to ERA vaccinal strain. Generally, from the result it can be concluded that the vaccine under trial can protect from the local virus, but potency should be determined with locally developed challenge virus which can guarantee efficacy of the vaccine under trial. This study strongly supports control and prevention of rabies through delivering high quality ant-rabies vaccine.

\section{Acknowledgement}

I would like to thank CDC Atlanta, USA for donation of fixed rabies virus strains I used during this study. I would also thank Prof. Yalemtsehay Mekonnen and Mr. Kelbessa Urga for their kind comment and advice during this research work. Finally I thank Ethiopian Public Health Institute for financial support for this research.

\section{References}

1. WHO (2005) World Health Organisation, WHO Technical Report Series, 93, Geneva. 1-87.

2. WHO (2010) Rabies: A neglected zoonotic disease.
3. Jemberu WT, Molla W, Almaw G, Alemu S (2013) Incidence of rabies in humans and domestic animals and people's awareness in North Gondar Zone, Ethiopia. PLoS Negl Trop Dis 7: e2216.

4. Bögel K, Motschwiller E (1986) Incidence of rabies and post-exposure treatment in developing countries. Bull World Health Organ 64: 883-887.

5. Deressa A, AliA, Beyene M, Selassie BN, Yimer E, et al. (2010) The status of rabies in Ethiopia: A retrospective record review. Ethiop. J Health Dev 24: 127-132.

6. Tefera G, Yimer E, Geyid A (2002) Endemic existence of rabies in Ethiopia. Ethiop Med J 40: 163-170.

7. Newayeselassie B, Yimer E, Abebe P, Bekele A, Zewdie B, et al. (2004) Occurrence of rabies in humans and animals in Addis Ababa in 2001-2002. Ethiop J Biol Sci 3: 59-67.

8. Horton DL, McElhinney LM, Marston DA, Wood JL, Russell CA, et al. (2010) Quantifying antigenic relationships among the lyssaviruses. J Virol 84: 11841-11848.

9. Wright E, Temperton NJ, Marston DA, McElhinney LM, Fooks AR, et al. (2008) Investigating antibody neutralization of lyssaviruses using lentiviral pseudotypes: a cross-species comparison. J Gen Virol 89: 2204-2213.

10. Badrane H, Bahloul C, Perrin P, Tordo N (2001) Evidence of two Lyssavirus phylogroups with distinct pathogenicity and immunogenicity. J Virol 75: 3268-3276.

11. Leslie MJ, Messenger S, Rohde RE, Smith J, Cheshier R, et al. (2006) Batassociated rabies virus in Skunks. Emerg Infect Dis 12: 1274-1277.

12. Mebatsion T, Cox JH, Frost JW (1992) Isolation and characterization of 115 street rabies virus isolates from Ethiopia by using monoclonal antibodies: identification of 2 isolates as Mokola and Lagos bat viruses. J Infect Dis 166: 972-977.

13. Branche R (1996) Vaccine for humans prepared in human diploid cells. 280-284.

14. Sacramento D, Badrane H, Bourhy H, Tordo N (1992) Molecular epidemiology of rabies virus in France: comparison with vaccine strains. J Gen Virol 73 : 1149-1158.

15. Hurisa B, Mengesha A, Newayesilassie B, Kerga S, Kebede G, et al. (2013) Production of cell culture based anti- rabies vaccine in Ethiopia. Proc. in Vaccinol 7: 2-7.

16. Hanlon CA, Kuzmin IV, Blanton JD, Weldon WC, Manangan JS, et al. (2005) Efficacy of rabies biologics against new lyssaviruses from Eurasia. Virus Res 111: 44-54.

17. Fekadu M, Shaddock JH, Sanderlin DW, Smith JS (1988) Efficacy of rabies vaccines against Duvenhage virus isolated from European house bats (Eptesicus serotinus), classic rabies and rabies-related viruses. Vaccine 6: 533-539.

18. Guo C, Wang C, Luo S, Zhu S, Li H, et al. (2014) The adaptation of a $\mathrm{CTN}-1$ rabies virus strain to high-titered growth in chick embryo cells for vaccine development. Virol J 11: 85. 